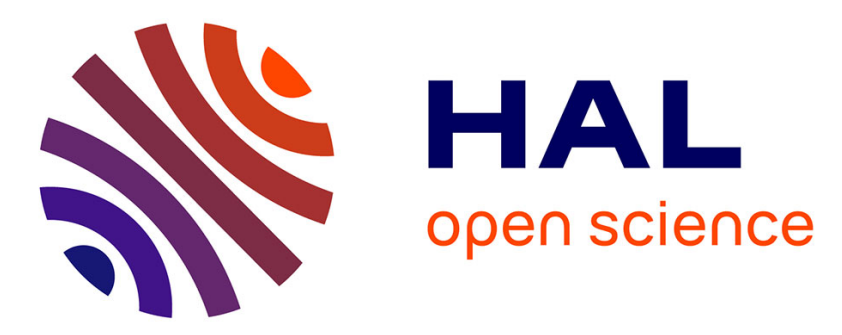

\title{
Identification of poroelastic constants of tight rocks from laboratory tests
}

\author{
Brice Lecampion, Andrei Constantinescu, Laurent Malinski
}

\section{To cite this version:}

Brice Lecampion, Andrei Constantinescu, Laurent Malinski. Identification of poroelastic constants of tight rocks from laboratory tests. International Journal of Geomechanics, 2006, 6, pp.201-208. 10.1061/(ASCE)1532-3641(2006)6:3(201) . hal-00111451

\section{HAL Id: hal-00111451 \\ https://hal.science/hal-00111451}

Submitted on 30 Jun 2019

HAL is a multi-disciplinary open access archive for the deposit and dissemination of scientific research documents, whether they are published or not. The documents may come from teaching and research institutions in France or abroad, or from public or private research centers.
L'archive ouverte pluridisciplinaire HAL, est destinée au dépôt et à la diffusion de documents scientifiques de niveau recherche, publiés ou non, émanant des établissements d'enseignement et de recherche français ou étrangers, des laboratoires publics ou privés. 


\title{
Identification of Poroelastic Constants of "Tight" Rocks from Laboratory Tests
}

\author{
Brice Lecampion ${ }^{1}$; Andrei Constantinescu²; and Laurent Malinsky ${ }^{3}$
}

\begin{abstract}
This paper discusses the identification of poroelastic constants and hydraulic conductivity from two transient laboratory tests performed on cylindrical core: drained isotropic confinement and pulse test. The combination of the two tests allows us to estimate all poroelastic parameters with the exception of the shear modulus. Despite the lack of analytical solutions for the fully coupled case, closed form solutions of the forward problems are obtained for a slender specimen. The validity of these solutions for a realistic aspect ratio of the core is assessed by a comparison with a finite element model. The identification problem is solved by minimizing a least square functional using an explicit gradient computed using the direct differentiation of the closed form solution and a Levenberg-Marquardt algorithm. The uniqueness of this inverse problem as well as the effect of noise on input data are fully discussed. The identification procedure is then applied to tests performed on a deep argillaceous rock (argillite of Meuse Haute-Marne).
\end{abstract}

Keywords: Poroelasticity; Permeability; Experimentation; Rocks; Laboratory tests; Hydraulic conductivity.

\section{Introduction}

This paper presents a method for the identification of porolastic constants of rocks with very low permeability, also denoted as "tight rocks." As the concept of very low permeability often depends on the intended applications, we specify here that "tight" rocks refer to materials having an intrinsic permeability $k$ under $10^{-20} \mathrm{~m}^{2}$. Typical applications are hydro-mechanical responses of rock masses for geological modeling, design of underground waste repositories, or others rock engineering projects involving large time scales.

These extreme values of permeability reach actually the physical possibilities of direct measurements. In these ranges, it is not possible to accurately measure the flow output of the sample or to control the inside pore pressure. It is known (Hart and Wang 2001), that classical steady state tests are not appropriate for this type of rocks. Therefore, several experimental methods and interpretation (Escoffier et al. 2001; Hart and Wang 2001) of the corresponding results have been developed to solve this problem.

Our main interest in this work lies, on the one hand, in interpreting the hydromechanical coupling during drained isotropic confinement and pulse tests performed on cylindrical specimens

${ }^{1}$ CSIRO Petroleum, Melbourne Office, PB 10, Clayton South VIC 3169, Australia. E-mail: brice.lecampion@csiro.au

${ }^{2}$ Lab. de Mécanique des Solides, CNRS, UMR 7649, Ecole Polytechnique, 91128 Palaiseau Cedex, France (corresponding author). E-mail: andrei.constantinescu@1ms.polytechnique.fr

${ }^{3}$ Lab. de Mécanique des Solides, CNRS, UMR 7649, Ecole Polytechnique, 91128 Palaiseau Cedex, France. E-mail: malinsky@ lms.polytechnique.fr and, on the other hand, in determining the poroelastic parameters from these experiments.

Both tests can be performed on rocks with extremely low hydraulic conductivity and are of transient nature spanning several days. As presented in the next sections, the measurements of radial and/or axial displacements during a drained isotropic confinement test permit us to identify the drained and undrained bulk moduli as well as a diffusivity coefficient $D$ lumping the hydraulic conductivity and the poroelastic parameters. The decrease of the reservoir pressure recorded during a pulse test is governed by two constitutive parameters: a lumped dimensionless coefficient $\chi$ of the poroelastic parameters and the same diffusivity coefficient $D$. A combined interpretation of these two tests should permit us to completely identify the poroelastic constants.

The main difficulty in the interpretation is the lack of simple closed-form solutions for such experiments (Adachi and Detournay 1997). Even in the framework of linear poroelasticity (Biot 1941; Detournay and Cheng 1993; Coussy 2004), no such solutions are known. In order to overcome this type of difficulty, it is common to interpret such transient tests with tabulated charts (Neuzil et al. 1981; Escoffier et al. 2001), sometimes neglecting the poroelastic coupling. As both tests are fully two dimensional, we shall first show that one-dimensional closed form solutions can be derived and applied providing the. specimen is slender enough. The validity of the one-dimensional solution for the twodimensional field is checked using fully coupled finite element simulations.

A complete identification method for the pulse and drained isotropic confining test is proposed. The parameter identification is here formulated as an inverse problem. The poroelastic constants are obtained by minimization of a cost functional measuring the discrepancy between computed predictions and measurements.

Finally, experiments performed on deep argillaceous rocks are analyzed using the proposed method and several details related to the modeling and the identification procedure are discussed. 

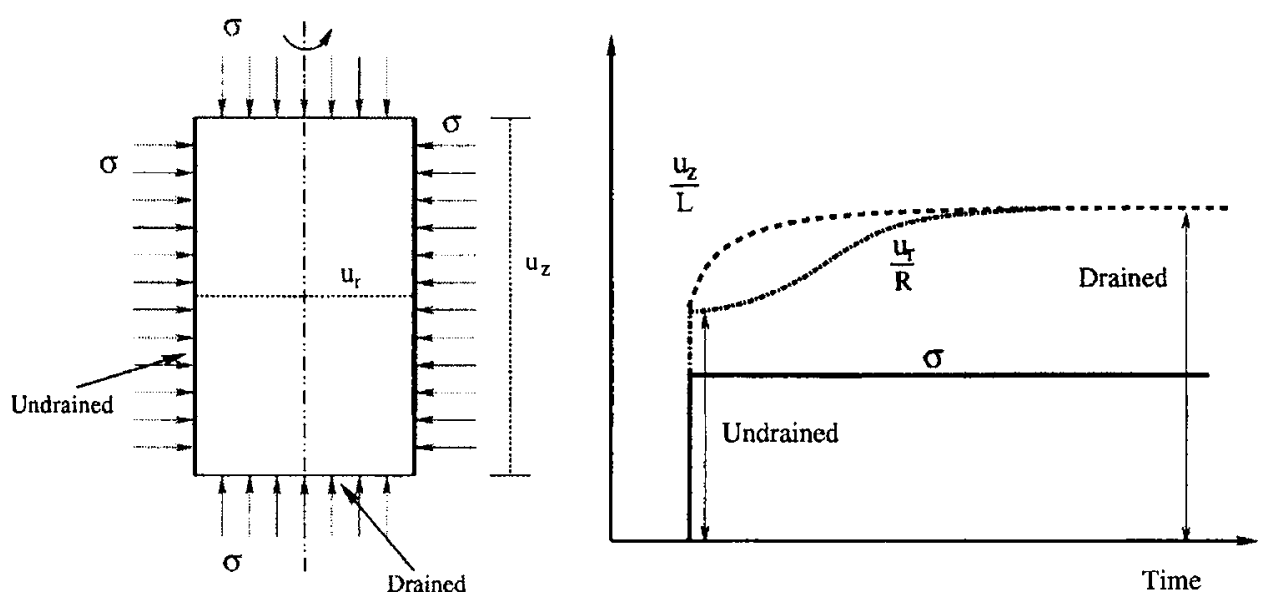

Fig. 1. Boundary conditions (left) and evolution of radial and axial displacements (right) for drained isotropic confinement test

\section{Tests Procedure}

Both the pulsed and the drained isotropic confining test are performed on a cylindrical core $[(r, z) \in[0, R] \times[0, L]$ with $R=1.8 \mathrm{~cm}, L=7.2 \mathrm{~cm}]$ under axisymmetric conditions.

The specimen is set between two porous end caps in a triaxial cell. The cell allows us to apply a confining pressure and the end caps permit us to impose the pore pressure at the top and bottom of the sample. The complete experimental setup is based on an extremely rigid servo-controlled frame. Precise details of the experimental setup can be found in Malinsky et al. (2002). However, let us simply highlight here several important aspects of the test facility:

1. The cylindrical surface of the specimen is lined with an impermeable jacket. The validity of this system for extremely low hydraulic conductivities rocks has been assessed by tests performed on steel cores with a rough cylindrical surface.

2. The compressibility of the pore pressure injection system has been reduced to a minimum.

3. The effect of thermal variations are corrected dynamically using a reference circuit.

4. The injected water is a reconstructed formation water.

5. Radial and axial displacements are measured via extensometers, while pressure-volume controllers are placed in the top and bottom hydraulic circuit.

Prior to the tests, the sample is resaturated under isotropic confinement for several weeks. After this preparation, the sample is in an homogeneous and isotropic hydromechanical state which can be considered as a reference initial state with regards to the linear theory of poroelasticity.

The drained isotropic confinement test consists of the sudden application at $t=0$ of an isotropic confining normal stress $\sigma$ on all the surfaces of the specimen. The surface tractions are given by

$$
\boldsymbol{\sigma} \cdot \boldsymbol{n}=\sigma_{n} H(t)
$$

where $\boldsymbol{\sigma}=$ stress tensor; and $\boldsymbol{n}=$ outside unit normal and $H(t)$ denotes the Heavyside step function. The cylindrical surface $(r=R)$ of the sample remains undrained, i.e., the fluid flux through the surface is zero

$$
\boldsymbol{q} \cdot \boldsymbol{n}=0
$$

while the top and bottom surfaces $(z=0, L)$ are drained (see Fig. 1)

$$
p=0
$$

where $p=$ pore pressure.

The recorded axial and radial displacements measure the evolution of the specimen from the undrained to the drained values.

The pulse test consists of the instantaneous injection of a water volume $V_{0}$ in a deformable reservoir of stiffness $C_{\text {res }}$ connected to the porous sample (see Fig. 2). This injection produces an instantaneous pressure increment $p_{0}$ in the reservoir. After a transient phase, the system reservoir specimen reaches an equilibrium state where the specimen pore pressure and the reservoir pressure are equal. The decrease of the reservoir pressure from $p_{0}$ toward its equilibrium value depends on both the poroelastic coefficients and the permeability of the specimen, as well as on the reservoir stiffness.

This transient method has been proposed first by Brace et al. (1968) for rocks with a negligible storage coefficient. The method has been extended afterward to other types of rocks by Hsieh et al. (1981) and is actually largely used (Zeynaldi-Andabily and Rahman 1995; Escoffier et al. 2001). However, in the case of a poroelastic coupling, special care has to be taken with respect to the real boundary condition. The cylindrical surface $(r=R)$ of the specimen is kept undrained during the test. The pore pressure on the boundary between the reservoir and the specimen, $\Gamma_{\text {res }}$, at the top and bottom surfaces, equals the fluid pressure in the reservoir $p_{\text {res }}(t)$ at all time instants. The evolution of the reservoir pressure

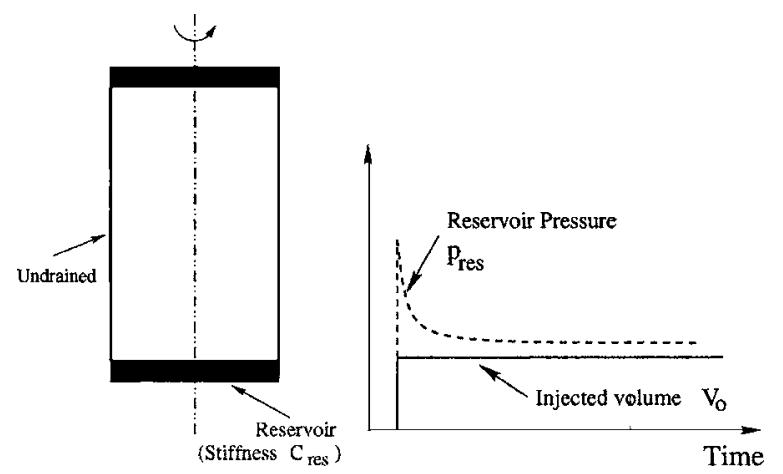

Fig. 2. Boundary conditions for pulse test (left) and typical decay of the reservoir pressure 


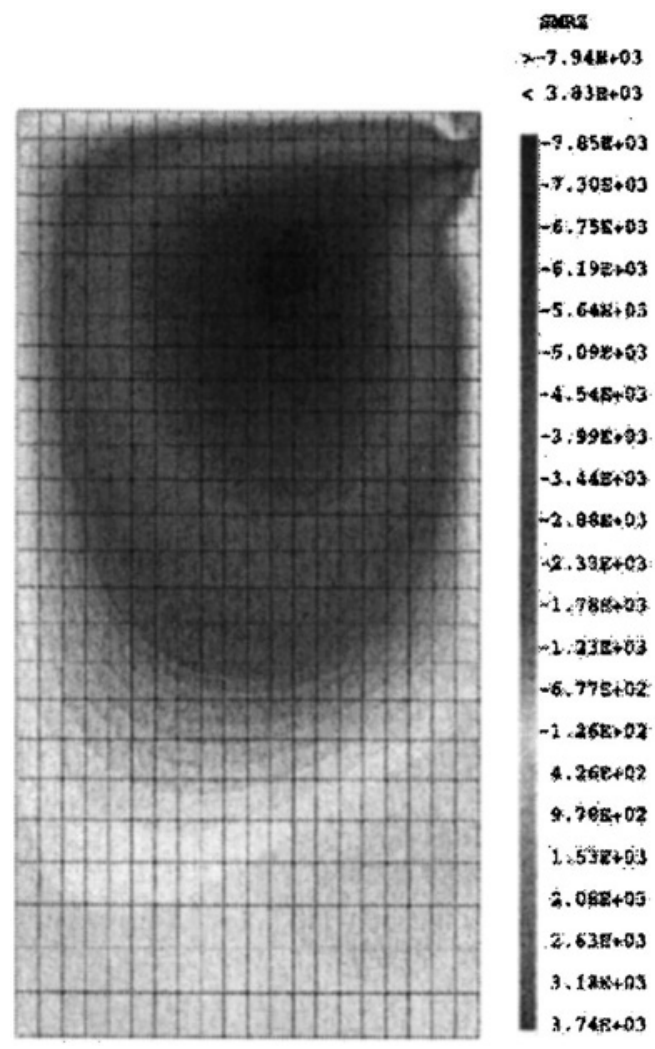

Fig. 3. Isovalue contours of shear component of stress tensor during transient phase of isotropic confining test. Finite-element model Cast3M, axisymmetry, poroelastic coupling.

is governed by the fluid volume balance. The balance is written, after taking into account the reservoir stiffness $C_{\text {res, }}$, as

$$
\int_{\Gamma_{\mathrm{res}}} \boldsymbol{q} \cdot \boldsymbol{n} d \boldsymbol{\Gamma}=\frac{1}{C_{\mathrm{res}}} \frac{d p_{\mathrm{res}}(t)}{d t}
$$

where $\boldsymbol{q}=$ fluid flux vector flowing in the specimen.

The confining stress $\sigma$ is kept constant during the test and is equal to the one of the reference state. However, as the pore pressure cannot be applied on a porous material without applying a mechanical pressure, the top and bottom sufaces $(z=0, L)$ of the samples are also subject to a compression

$$
\boldsymbol{\sigma}(t) \cdot \boldsymbol{n}=-p_{\text {res }}(t) \boldsymbol{n}
$$

If this last boundary condition is ignored, then the Skempton effect will be equally ignored. As a consequence the estimation of the poroelastic parameters will be incorrect.

\section{Modeling and Parameter Identification}

\section{Closed Form Solutions}

The poroelastic fields describing the tests are fully twodimensional. As an illustration of this fact, we have displayed spatial distribution of the shear stress component during a drained isotropic confinement test in Fig. 3.

As explained by Adachi and Detournay (1997), the poroelastic coupling does not allow to obtain a closed form solution for these particular configurations. This stems essentially from the hydromechanical boundary conditions on the cylindrical surface: no

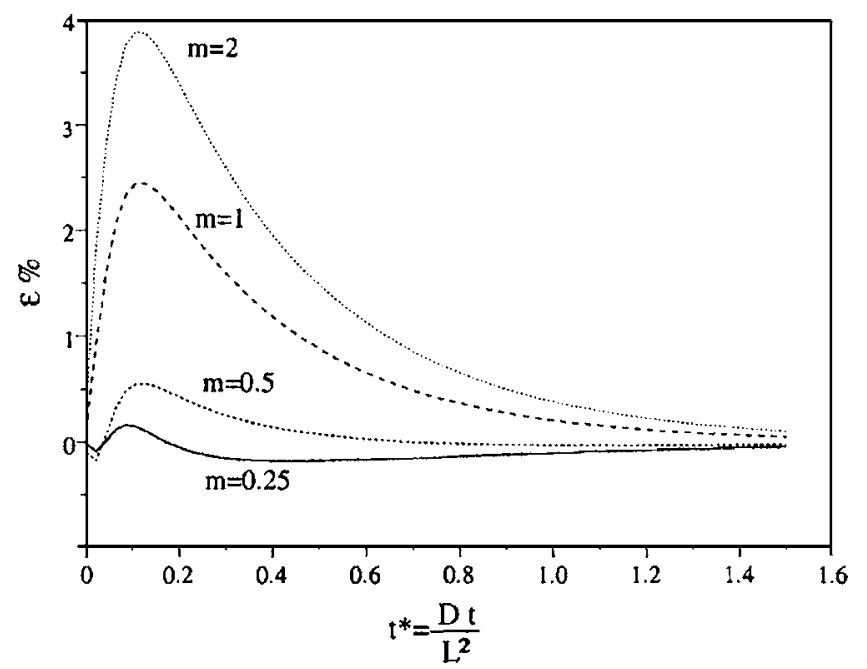

Fig. 4. Relative error on axial strain between closed form and coupled finite-element solution for different aspect ratios $m=R / L$

hydraulic flux and an applied radial stress. In spite of the apparent simplicity of these boundary conditions, the theory of linear poroelasticity yields to nonhomogeneous hydromechanical fields in the core during the transient phase. It has also been shown (Adachi and Detournay 1997), that for the limiting case of a very slender specimen $(m=R / L \ll 1)$, the continuity equation governing the pore pressure evolution in the sample, and the balance equation uncouple. In the limiting case, stress and pore pressure fields are homogeneous in the sample, which finally translates into a one-dimensional closed-form solution. The expressions of the closed form solutions for both tests are given in the Appendix.

We have first addressed the validity of the slender assumption in the case of our specimen, which has a given aspect ratio $m=R / L=0.25$. A series of axisymmetric finite element simulations using the object oriented finite element code Cast3M (2005) have been performed for different aspect ratios in order to investigate the hypothesis of slender specimen $(m=R / L \ll 1)$.

We have analyzed the relative error between the axisymmetric FEM and one-dimensional solution for different quantities measured during the experiments. As a typical example in Fig. 4, we have plotted for the case of the drained isotropic confinement test the relative error on the vertical displacements for a series of different aspect ratios. We remark that the error is only significant during the transient phase and decreases with the value of the aspect ratio $m=R / L$. Similar patterns have also been observed for the pulse test. We also note that computations for different values of the shear modulus did not show a major influence on this relative error.

As a consequence, we can conclude that for the aspect ratio of the specimen used in our experiments $(m=0.25)$, the coupled effect is negligible. The relative error $\epsilon$ on the axial strain is at most of $1 \%$ and will therefore permit the usage of the one-dimensional closed-form solution in the sequel. This fact simplifies the computional effort for the identification process, saving both programming and computation time compared to a finite element solution.

\section{Parameter Identification}

The question of the parameter identification problem is: "How to invert the displacements and the reservoir pressure measured during the tests in order to obtain an estimation of some poroelastic parameters?" 
Table 1. Identifiable Coefficients and Corresponding Tests

\begin{tabular}{lcl}
\hline Coefficient & Dimensions & Test \\
\hline$K, K_{u}$ drained and & {$\left[\mathrm{ML}^{-1} \mathrm{~T}^{-2}\right]$} & $\begin{array}{l}\text { Drained isotropic } \\
\text { confinement } \\
\text { undrained bulk moduli }\end{array}$ \\
$D=\frac{k}{\mu_{f}} \frac{M K}{K_{n}}$ diffusivity & {$\left[\mathrm{L}^{2} \mathrm{~T}^{-1}\right]$} & $\begin{array}{l}\text { Drained isotropic } \\
\text { confinement/pulse test }\end{array}$ \\
$\chi=\frac{M K}{C_{\text {res }} K_{u} \pi R^{2} L}\left(1-\frac{B}{3}\right)$ & {$[\cdot]$} & Pulse test \\
\hline
\end{tabular}

Note: $B=$ Skempton's Coefficients; $M=$ Biot's modulus; $k=$ intrinsic permeability of the sample; $C_{\text {res }}=$ reservoir stiffness; and $\mu_{f}=$ water viscosity.

A direct interpretation of the solutions of both test shows that different parameters are related to one or the other solutions (see Table 1). The drained $K$, the undrained $K_{u}$ bulk moduli, and the diffusivity coefficient $D$ [expression (3)] can be identified from the recorded evolution of the axial $\left[u_{z}(z=L, t)\right]$ and/or radial $\left[u_{r}(z=L / 2, t)\right]$ displacements during the drained isotropic confinement test [see Eq. (5) in the Appendix]. From the pulse test, the dimensionless parameters $\chi$ [expression (6)] and the diffusivity coefficient $D$ can be identified from the recorded reservoir pressure decay $p_{\text {res }}(t)$ [see Eq. (5) in the Appendix].

A close inspection of the expressions for displacements and pressure show that they involve infinite series and Laplace transforms. As a consequence, we do not possess a simple expression relating directly the unknown parameters to the measurements. The proposed solution of the identification problem will be therefore based on the minimization of a least square cost functional measuring the distance between predictions and measurements. The minimization of this functional is performed using a Levenberg-Marquardt gradient based algorithm (see Gill et al. 1982 for details)

One of the important technical requirements of gradient based optimization algorithms is the accuracy of the gradient component. In the case where the direct problems are solved numerically, special numerical techniques such as the adjoint state method and the direct differentiation needs to be used in order to obtain precise estimation of the gradient (see Lecampion and Constantinescu 2005 for a derivation of the poroelastic case). However, here the knowledge of the closed form solutions permitted the computation of the exact gradients of the solution in the directions of the unknown parameters, the so-called sensitivities

$$
\frac{d u}{d K} \quad \frac{d u}{d K_{u}}, \cdots \frac{d p}{d M}, \quad \frac{d p}{d \chi}
$$

The sensitivities of the axial displacement with respect to different parameters in the case of the isotropic confinement test are displayed in Fig. 5. We should note that the experimental response contains information on the undrained modulus $K$ at early times, but the information fades later. At large times, the displacement depends, as expected, mainly on the drained modulus. The sensitivity on the diffusivity coefficient is maximum during the transient phase. Similar patterns can be observed for the pulse test and could be used for fine tuning of the identification algorithm.

We recall that the estimation of unknowns parameters from measurements is an inverse problem and that it may not have a unique solution (Vogel 2002). However, for both tests considered here, algebraic manipulations of the closed form solutions permit us to show that uniqueness and stability are assured at least for the ideal mathematical problem (see Lecampion 2002 for details).

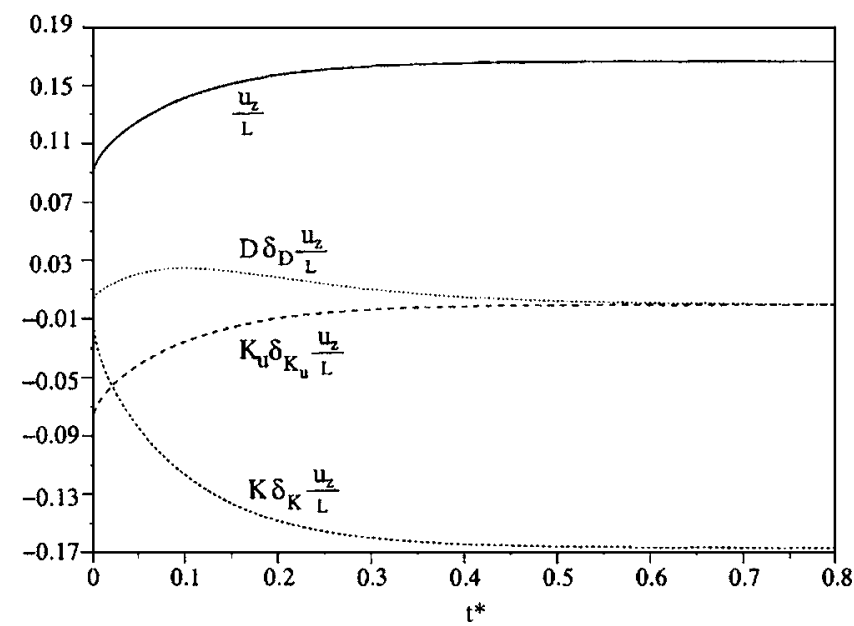

Fig. 5. Dimensionless total axial displacement $u_{z} / L$ and associated sensitivity coefficient on $K, K_{u}$, and $D$ during drained isotropic confinement test

In the case of real data, where the poroelastic model may not be adequate, the identification can prove to be difficult and unstable. The validity of the results can be simply assessed by running the minimization algorithm with several different starting points for the initial guess of the parameters.

\section{Results and Discussion}

The drained isotropic confinement and the pulse test have been performed on several argillaceous rock samples. The tested argilite belongs to the Callovo-Oxfordian formation of Meuse HauteMarne (Bure, France) (Malinsky et al. 2002). The samples are taken from cores at a depth of about $500 \mathrm{~m}$, and its properties of this rock are extensively described in ANDRA, Direction Scientifique (1999). In this section, we present some of the identification results for the poroelastic constants obtained using the tests and the identification procedure described previously.

\section{Drained Isotropic Confinement}

Fig. 6 and 7, display the measured and fitted responses of an argilite sample submitted to a drained isotropic confinement. As a first remark, one can note the high level of anisotropy between the axial and radial deformation. The identification performed using only the axial displacement of the isotropic drained consolidation on an argilite sample gives a perfect fit (Fig. 6). For different initial guesses the same set of identified parameters is obtained

$$
K=1.04 \mathrm{GPa} ; \quad K_{u}=9.82 \mathrm{GPa} ; \quad D=4.02 \times 10^{-9} \mathrm{~m}^{2} \mathrm{~s}^{-1}
$$

The identification performed using only the radial displacement recorded at the middle of the specimen yields the following values of the parameters:

$$
K=2.12 \mathrm{GPa} ; \quad K_{u}=16.9 \mathrm{GPa} ; \quad D=1.74 \times 10^{-9} \mathrm{~m}^{2} \mathrm{~s}^{-1}
$$

Comparing the experimental and computed responses one can observe a certain misfit (see Fig. 7). A close inspection of this figure shows on the one hand that the measured radial displacement has not reached its asymptotic value after 15 days, whether the computed one already tends to its asymptotic value. On the other hand, at early times the measured initial delay due to the 


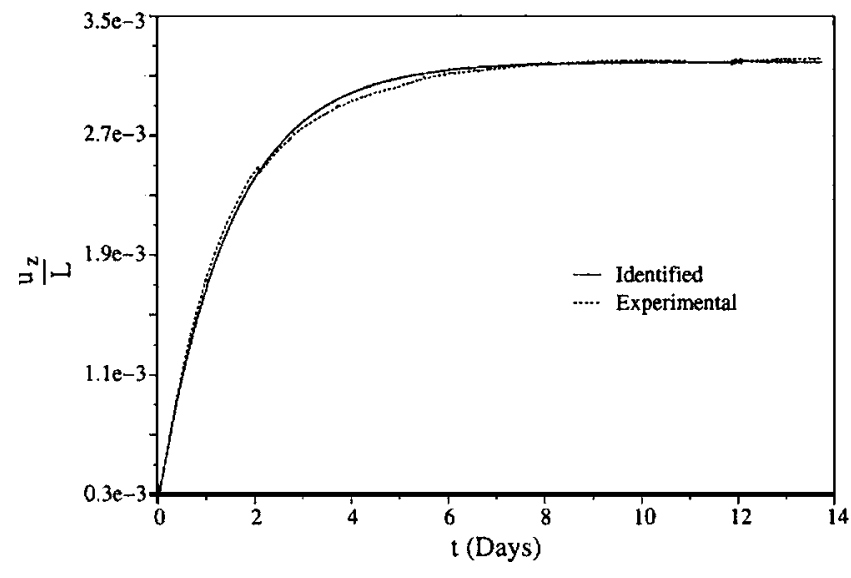

Fig. 6. Experimental and identified response (axial strain) to isotropic confinement test performed on argilite of Meuse Haute-Marne

diffusion process is rather small when compared to the computed one. In this case, using the knowledge of the uniqueness result for the identification problem one can state that the mismatch is not an artifact of the identification. The linear poroelastic model is probably not suited to represent the material behavior. This has later been confirmed by swelling observed on the same sample.

The anisotropy is clearly straightforward when looking at the parameters estimated with either axial or radial displacement. This anisotropy can be explained by the high level of damage of the sample, which most probably occurred during sample preparation.

Knowing $K, K_{u}$, and $D$, it is possible to compute the intrinsic permeability assuming reasonable value for Biot's modulus $M$. Table 2 presents the results for different values of $M$. An intrinsic permeability on the order of magnitude of $1 \times 10^{-21} \mathrm{~m}^{2}$ matches the expected values for this argilite.

\section{Pulse Test}

The identification method has also been applied to pulse tests. The experimental as well as the identified response are displayed in Fig. 8. Table 3 presents the identified parameters as well as the value of the cost functional for different initial guesses. The best fit has been obtained for the following set of values:

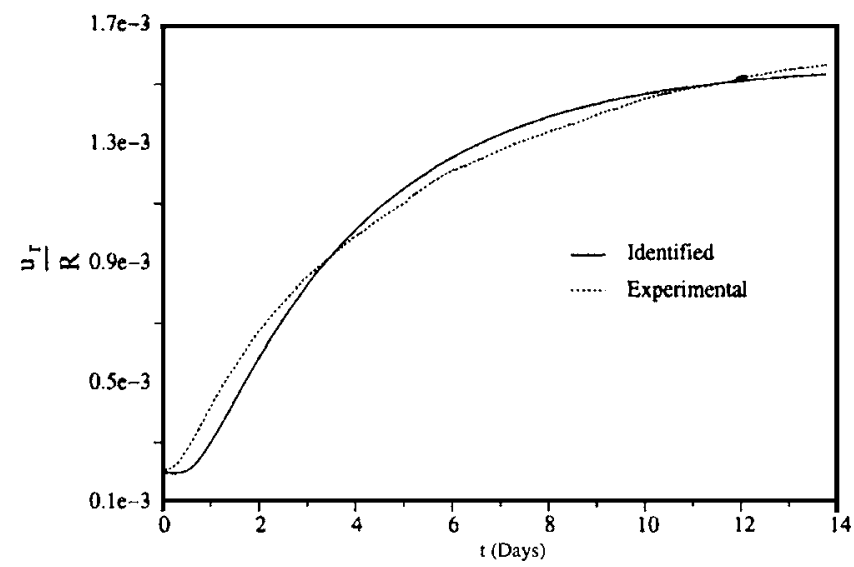

Fig. 7. Radial strain (isotropic confinement)
Table 2. Estimation of Intrinsic Permeability for Different Values of Biot Modulus with $K=1.04 \mathrm{GPa}, K_{u}=9.82 \mathrm{GPa}$, and $D=4.02 \times 10^{-9} \mathrm{~m}^{2} \mathrm{~s}^{-1}$ (Identification Performed on Axial Displacement)

\begin{tabular}{lc}
\hline $\mathrm{M}$ & $k$ \\
$(\mathrm{GPa})$ & $\left(\mathrm{m}^{2}\right)$ \\
\hline 5 & $7.5 \times 10^{-21}$ \\
10 & $3.7 \times 10^{-21}$ \\
15 & $2.5 \times 10^{-21}$ \\
\hline
\end{tabular}

$$
\chi=0.653 ; \quad D=1.65 \times 10^{-9} \mathrm{~m}^{2} \mathrm{~s}^{-1}
$$

However, the match between the measured and computed responses is far from perfect. As before, taking into account the stated uniqueness of the identification procedure, one can conclude that this relative misfit is attributed to the poroelastic model, which is not able to capture the complex behavior of this rock sample.

At this point it is important to note that the exact value of the reservoir stiffness $C_{\text {res }}$ intervenes directly in the identification process and has to be known with precision in order to extract accurate values of the poroelastic parameters. The reservoir stiffness can be estimated by additional tests on the reservoir. Unfortunately, in our case, this estimation was far from perfect.

In Table 5, we have displayed the value of the coefficient $M K / K_{\mathrm{u}}$ and that of the intrinsic permeability $k$ for a range of acceptable values of the reservoir stiffness $C_{\text {res }}$. We should note that all the obtained values are in a standard range for an argilite.

\section{Discussion}

As a first conclusion, we note that all the identified values of the parameters lies in the physical range known from the literature (see Table 4). Previous laboratory experiments (Vincké et al. 1998; Escoffier et al. 2001) suggest a value around $10^{-21} \mathrm{~m}^{2}$ for the intrinsic permeability of these rocks. The value that we can deduce (Tables 3 and 5) tends to be slightly lower: $10^{-22} \mathrm{~m}^{2}$. The values of the elastic undrained moduli found are around known values. The drained values obtained here are lower than the one reported previously (Escofier et al. 2001). This may be partly due to the high level of damage to the sample observed after the test.

Despite the reported misfit between model predictions and measurements we can conclude that the first order response of the

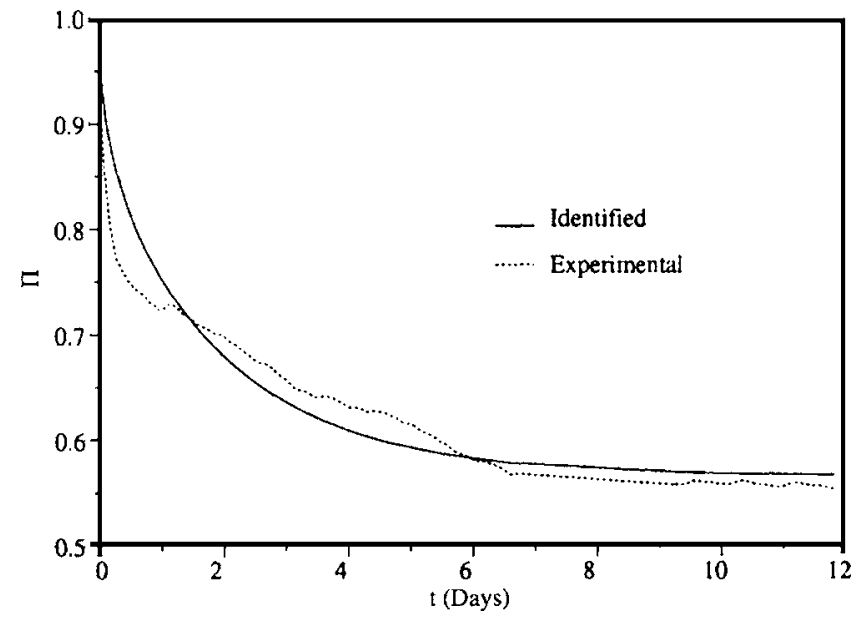

Fig. 8. Pulse test on argilite from Meuse Haute-Marne 
Table 3. Pulse Test on Argilite. Results of Identification for Different Initial Guesses

\begin{tabular}{llll}
\hline & \multicolumn{1}{c}{$\chi$} & \multicolumn{1}{c}{$D\left(\mathrm{~m}^{2} \mathrm{~s}^{-1}\right)$} & $\mathcal{J}$ \\
\hline Initial & 0.8 & $5.184 \times 10^{-9}$ & 0.21 \\
Final & 0.6531 & $1.65162 \times 10^{-9}$ & 0.09084 \\
Initial & 1.8 & $5.184 \times 10^{-8}$ & 2.872 \\
Final & 0.653 & $1.6537 \times 10^{-9}$ & 0.09084 \\
Initial & 0.1 & $7.776 \times 10^{-10}$ & 16.65 \\
Final & 0.6537 & $1.65629 \times 10^{-9}$ & 0.0906 \\
\hline
\end{tabular}

rock to the experiments is clearly of linear poroelastic nature. The knowledge of the uniqueness of the identification for perfectly poroelastic materials is practically important as it can quantify the deviation of the specimen from a purely poroelastic behavior. Such information can guide further modeling of the hydromechanical behavior of such rocks.

One should not be mislead by the obtained experimental results. The technical difficulties of performing tests on such argillaceous materials are enormous and the values of the parameters should be taken with precaution as preliminary estimations. Several experimental and modeling issues regarding the chemically reactive and swelling behavior of this argilite should also be investigated in more details.

An important advantage has been obtained by performing the two tests, drained isotropic confinement and pulse test, together. By combining the identified coefficients from the two tests $\left(K, K_{u}, D\right.$, and $\left.\chi\right)$, it is possible to estimate all the poroelastic parameters and the permeability with only the exception of the shear modulus. Moreover, as the diffusivity coefficient $D$ is estimated twice in two different ways, it ensures the robustness of the estimation. Complete results of this procedure could not be displayed here due to confidentiality agreements.

Finally, we note that the closed form solution used for the pulse test is similar to the one proposed by Hsieh et al. (1981) for the uncoupled case. The difference is that we explicitly take into account the presence of a mechanical pressure on the reservoir surface. The presence of this boundary condition largely affects the identified parameters in the coupled case.

\section{Conclusions}

In this paper, we have discussed the identification of several poroelastic parameters from drained isotropic confinement and pulse tests. We have shown that for specimens with an aspect ratio $R / L \ll 0.25$, we can use the one-dimensional closed form solutions obtained for a slender specimen. This permits us to speed up the identification procedure. The proposed identification method has been shown to provide robust and efficient solutions to this

Table 4. Summary of Identified Values of Parameters for Collovo-Oxfordian Argilite

\begin{tabular}{lcc}
\hline Coefficient & Value & Test \\
\hline$K$ & $1-2 \mathrm{GPa}$ & Drained confinement \\
$K_{u}$ & $9-16 \mathrm{GPa}$ & Drained confinement \\
$D$ & $1.7-410^{-9} \mathrm{~m}^{2} \mathrm{~s}^{-1}$ & Drained confinement \\
$D$ & $3.5 \times 10^{-9} \mathrm{~m}^{2} \mathrm{~s}^{-1}$ & Pulse test \\
$X$ & 0.653 & Pulse test \\
\hline
\end{tabular}

Table 5. Estimation of Coefficient $M K / k_{u}$ and Intrinsic Permeability for Different Values of Reservoir Compressibility and Skempton's Coefficient $\left(\chi=0.653, D=3.5310^{-9} \mathrm{~m}^{2} \mathrm{~s}^{-1}\right)$

\begin{tabular}{lccl}
\hline $\mathrm{B}$ & $C_{\text {res }}\left(\mathrm{MPa} \mathrm{m}^{-3}\right)$ & $\frac{M K}{K_{u}}(\mathrm{MPa})$ & \multicolumn{1}{c}{$k\left(\mathrm{~m}^{2}\right)$} \\
\hline 0.0 & $8 \times 10^{4}$ & 15.536 & $2.27 \times 10^{-22}$ \\
0.0 & $4 \times 10^{4}$ & 7.76 & $4.54 \times 10^{-22}$ \\
0.0 & $1.33 \times 10^{4}$ & 2.58 & $2.654 \times 10^{-21}$ \\
1.0 & $8 \times 10^{4}$ & 23.4 & $1.5 \times 10^{-22}$ \\
1.0 & $4 \times 10^{4}$ & 11.7 & $3.01 \times 10^{-22}$ \\
1.0 & $1.33 \times 10^{4}$ & 3.9 & $9.07 \times 10^{-22}$ \\
\hline
\end{tabular}

inverse problem. Note that the induced mechanical loading during a pulse test greatly affects the estimated value of the poroelastic parameter as it induces a Skempton's effect.

The identified values of the constitutive parameters for the argilite of Meuse Haute-Marne are within the expected range. The partial mismatches between the poroelastic model and the experiment show that probably other physical phenomena (swelling, chemical reaction ....) should also be taken into account.

We can conclude that the combination of the drained isotropic confinement and the pulse test permits a complete identification of the poroelastic parameters and hydraulic conductivity with the exception of the shear modulus. The methodology presented here can be extended to other porous materials such as tight sandstone or granite.

\section{Acknowledgments}

The authors would like to thank Professor E. Detournay and Dr. J. I. Adachi for the benefit of useful discussions. This research was funded by the French Agency for Radioactive Waste Management (ANDRA) through a Ph.D. Grant for B.L. The experimental campaign was run for ANDRA under Contract No. ANDRA 017079-Contract-cadre AQL. The experimental tests were performed at G3S, Ecole Poly Y technique, France by Serge Chanchole and François Coste.

\section{Appendix. Closed-Form Solutions for Slender Case}

For the slender case $(R / L \ll 1)$, the problems are one dimensional and the continuity and balance equations uncouple. The diffusion equation governing the pore pressure now reduces to

$$
\frac{\partial p}{\partial t}-D \frac{\partial^{2} p}{\partial z^{2}}=-\frac{B}{3} \frac{d}{d t}[\operatorname{tr}(\boldsymbol{\sigma})]
$$

where $\operatorname{tr}(\boldsymbol{\sigma})=\sigma_{k k}=$ volumetric part of the stress tensor; and $B[\cdot]=$ Skempton dimensionless coefficient (between brackets $[\cdot]$ we indicate the physical dimensions of the parameters). The diffusivity $D\left[\mathrm{~L}^{2} \mathrm{~T}^{-1}\right]$ is given by (Detournay and Cheng 1993; Wang 2000)

$$
D=M \frac{k}{\mu_{f}} \frac{K}{K_{u}}
$$

where $k\left[\mathrm{~L}^{2}\right]=$ intrinsic permeability; $\mu_{f}\left[\mathrm{ML}^{-1} \mathrm{~T}^{-2}\right]=$ fluid viscosity; $M\left[\mathrm{ML}^{-1} \mathrm{~T}^{-2}\right]=$ Biot modulus; $K\left[\mathrm{ML}^{-1} \mathrm{~T}^{-2}\right]=$ drained bulk modulus; and $K_{u}\left[\mathrm{ML}^{-1} \mathrm{~T}^{-2}\right]=$ undrained bulk modulus. 
The right hand side of the continuity equation is different for each test

$$
\frac{d \operatorname{tr}(\boldsymbol{\sigma})}{d t}= \begin{cases}3 \sigma_{n} \delta(t) & \text { for the isotropic confinement } \\ \frac{d p_{\text {res }}}{d t} & \text { for the pulse test }\end{cases}
$$

where $\delta(t)=$ Dirac distribution.

We use a similar set of dimensionless variables for both tests

$$
\begin{gathered}
t^{*}=\frac{D t}{L^{2}}, \quad z^{*}=\frac{z}{L} \\
\Pi= \begin{cases}\frac{p}{\sigma} & \text { for the isotropic confinement test } \\
\frac{p}{p_{0}} & \text { for the pulse test }\end{cases}
\end{gathered}
$$

\section{Drained Isotropic Confinement Test}

The boundary conditions are homogeneous

$$
\begin{gathered}
\Pi\left(z^{*}=L, t\right)=\Pi\left(z^{*}=0, t\right)=0 \\
\Pi\left(z, t^{*}=0\right)=0
\end{gathered}
$$

The dimensionless pore pressure is easily obtained from the solution of the diffusion equation in a bounded one-dimensional domain with an instantaneous volumetric source term at $t^{*}=0$ (Carslaw and Jaeger 1959)

$$
\Pi\left(z^{*}, t^{*}\right)=-B \mathcal{K}\left(z^{*}, t^{*}\right)
$$

with $\mathcal{K}(z, t)$

$$
\mathcal{K}\left(z^{*}, t^{*}\right)=\frac{2}{\pi} \sum_{n=1}^{\infty} \exp \left(-n^{2} \pi^{2} t^{*}\right) \sin \left(n \pi z^{*}\right)\left(\frac{1}{n}-\frac{\cos (n \pi)}{n}\right)
$$

From the linear poroelastic constitutive equations in cylindrical coordinates (Biot 1941; Detournay and Cheng 1993; Wang 2000), one obtains the overall axial, and the radial displacement at the middle of the specimen. These displacements are recorded during an experimental test

$$
\begin{gathered}
\frac{u_{z}\left(z^{*}=1, t^{*}\right)}{L}=\frac{\sigma}{3 K}\left[1-\frac{K_{u}-K}{K_{u}} \mathcal{I}\left(1, t^{*}\right)\right] \\
\frac{u_{r}\left(z^{*}=1 / 2, t^{*}\right)}{R}=\frac{\sigma}{3 K}\left[1-\frac{K_{u}-K}{K_{u}} \mathcal{K}\left(1 / 2, t^{*}\right)\right]
\end{gathered}
$$

where $\mathcal{I}\left(l, t^{*}\right)=\int_{0}^{1} \mathcal{K}\left(\xi, t^{*}\right) d \xi$ is given by the following series:

$$
\mathcal{I}\left(1, t^{*}\right)=\frac{2}{\pi^{2}} \sum_{n=1}^{\infty} \exp \left(-n^{2} \pi^{2} t^{*}\right)\left(\frac{1}{n}-\frac{\cos n \pi}{n}\right)^{2}
$$

Series (4) and (5) are precisely estimated even for early times $\left(t^{*} \approx 0.01\right)$ when keeping the first ten terms of the series.

\section{Pulse Test}

This solution is similar to the classical one given by Hsieh et al. (1981) with the difference that we take into account the effect of the mechanical loading on the specimen due to the reservoir pressure.

The initial and boundary conditions (1) give the following additional equations to the pressure equation (2):

$$
\begin{gathered}
\Pi\left[z^{*}=(0,1), t^{*}=0\right]=1 \\
\Pi\left(z^{*}, t^{*}=0\right)=0 \\
\gamma \frac{d \Pi}{d t^{*}}-\frac{\partial \Pi}{\partial z^{*}}=0 \quad \text { in } z^{*}=0,1
\end{gathered}
$$

Another dimensionless parameter related to the constitutive parameters and the reservoir compressibility is introduced

$$
\gamma=\frac{M K}{C_{r} K_{n} \pi R^{2} L}
$$

The Laplace transform of the solution $\bar{\Pi}$ is given in the Laplace domain by

$$
\bar{\Pi}\left(z^{*}, s\right)=\frac{\chi \cosh \left[\sqrt{s}\left(1 / 2-z^{*}\right)\right]}{\chi s \cosh \left(\frac{\sqrt{s}}{2}\right)+\sqrt{s} \sinh \left(\frac{\sqrt{s}}{2}\right)}
$$

with

$$
\chi=\gamma\left(1-\frac{B}{3}\right)=\frac{K M}{K_{u} C_{\mathrm{res}} \pi R^{2} L}\left[1-\frac{\sqrt{M\left(K_{u}-K\right)}}{3 K_{u}}\right]
$$

Note that if we do not take into account the mechanical pressure, the solution has a similar form but with $\chi=\gamma$ : the Skempton's effect is ignored. No simple analytical expression of the inverse Laplace transform exists for expression (5). The inversion can be performed numerically using Stehfest algorithm, for example (Cheng et al. 1994).

\section{References}

Adachi, J. I., and Detournay, E. (1997). "A poroelastic solution of the oscillating pore pressure method to measure permeabilities of "tight" rocks." Int. J. Rock Mech. Min. Sci., 34(3-4).

ANDRA, Direction Scientifique (1999). "Reférentiel géologique du site de l'est." Rep. ANDRA ARP ADS 99-0 05, France.

Biot, M. A. (1941). "General theory of three-dimensional consolidation." J. Appl. Phys., 12, 155-164.

Brace, W. F., Walsh, J. B., and Frangos, W. T. (1968). "Permeability of granite under high pressure." J. Geophys. Res., 73(6), 2225-2236.

Carslaw, H. S., and Jaeger, J. C. (1959). Conduction of heat in solids, Oxford Science Publications, Oxford, U.K.

CAST3M. (2005). 〈www.cast3m.cea.fr〉.

Cheng, A. H.-D., Sidauruk, P., and Abousleiman, Y. (1994). "Approximate inversion of the Laplace transform." Mathematica, 4(2), 76-82.

Coussy, O. (2004). Poromechanics, Wiley, New York.

Detournay, E., and Cheng, A. H.D. (1993). Fundamentals of poroelasticity, in comprehensive rocks engineering, Vol. 2, Chap. 5, Pergamon, London, 113-171.

Escoffier, S., Homand, F., Giraud, A., and Su, K. (2001). "Mesure de la perméabilité et du coefficient de Biot dans les argilites de l'est (in French)." Proc., 15th Congrès Français de Mécanique, Nancy, France. 
Gill, P. E., Murray, W., and Wright, M. H. (1982). Practical optimization, Academic, New York.

Hart, D. J., and Wang, H. F. (2001). "A single test method for determination of poroelastic constants and flow parameters in rocks with low hydraulic conductivities." Int. J. Rock Mech. Min. Sci., 38, 577-583.

Hsieh, P. A., Neuzil, C. E., Bredehoeft, J. D., and Silliman, S. E. (1981). "Transient laboratory method for determining the hydraulic properties of tight rocks. I: Theory." Int. J. Rock Mech. Min. Sci., 18, 245-252.

Lecampion, B. (2002). "Sur l'identification des paramètres des lois de comportements des roches argileuses." Ph.D. thesis, Ecole Polytechnique, Palaiseau, France (in French).

Lecampion, B., and Constantinescu, A. (2005). "Sensitivity analysis for parameter identification in quasi-static poroelasticity." Int. J. Numer. Analyt. Meth. Geomech., 29, 163-185.

Malinsky, L., Chanchole, S., and Coste, F. (2002). "Identification of poroelastic constants of deep argillaceous rocks. I: Experimental set up and qualitative analysis." Proc., 2nd Biot Conf. on Poromechanics, Grenoble, France, J. L. Auriault et al., ed., Balkema, Rotterdam, The Netherlands.

Neuzil, C. E., Cooley, C., Silliman, S. E., Bredehoeft, J. D., and Hsieh, P. A. (1981). "Transient laboratory method for determining the hydraulic properties of tight rocks. II: Application.” Int. J. Rock Mech. Min. Sci., 18, 253-258.

Vincké, O., Longuemarre, P., Boutéca, M., and Deflandre, J. P. (1998). "Investigation of the poromechanical behavior of shales in the elastic domain." SPE/ISRM 47589, 515-520.

Vogel, C. (2002). Computational methods for inverse problems, SIAM.

Wang, H. F., (2000). Theory of linear poroelasticity with applications to geomechanics and hydrogeology, Princeton University Press, N.J.

Zeynaldi-Andabily, E. M., and Rahman, S. S. (1995). "Measurement of permeability of tight rocks.” Meas. Sci. Technol., 6, 1519-1527. 